Also, the testicular teratoma ${ }^{5}$ showing complete chondrosarcomatous differentiation is an interesting case although the histology shown in the manuscript does not clearly correspond to conventional chondrosarcoma but rather shows additionally undifferentiated mesenchyme. Again, a reinvestigation of the case with currently available tools would be worthwhile, which would allow identification and classification of chondrocyte differentiation. . $^{6}$

Overall, clearly single (ie, very exceptional) cases may exist that represents extreme variants of neoplastic differentiation spectra. Whether this includes chondroma-identical pleomorphic adenoma and chondrosarcoma-identical chondroid chordoma requires further investigations. However, one should not forget, in practice, that these "extreme" cases are very rare-if they exist at all. Thus, particularly in cases in which the neoplastic counterparts (ie, chondrosarcoma for chondroid chordomas) are rather common, one should be very reluctant not to take these as a first diagnostic choice, even though this might, in a very exceptional case, be wrong.

\section{University of Erlangen-Nurnberg}

Erlangen, Germany

Thomas Aigner

\section{References}

1. Gottschalk D, Fehn M, Patt S, Saeger W, Kirchner T, Aigner T: Matrix gene expression analysis and cellular phenotyping in chordoma reveals focal differentiation pattern of neoplastic cells mimicking nucleus pulposus development. Am J Pathol 2001, 158:1571-1578

2. Gusterson BA, Lucas RB, Ormerod MG: Distribution of epithelial membrane antigen in benign and malignant lesions of the salivary gland. Virchows Arch 1982, 397:227-233

3. Aigner T, Neureiter D, Völker U, Belke J, Kirchner T: Epithelial-mesenchymal differentiation and extracellular matrix gene expression in pleomorphic adenomas of the salivary glands. J Pathol 1998, 186: $178-185$

4. Stead RH, Qizilbash AH, Kontozoglou T, Daya AD, Riddell RH: An immunohistochemical study of pleomorphic adenomas of the salivary glands. Hum Pathol 1988, 19:32-40

5. Füzesi L, Rixen H, Kirschner-Hermanns R: Cytogenetic findings in a metastasizing primary testicular chondrosarcoma. Am J Surg Pathol 1993, 17:738-742

6. Aigner T, Dertinger S, Vornehm SI, Dudhia J, von der Mark K, Kirchner $\mathrm{T}$ : Phenotypic diversity of neoplastic chondrocytes and extracellular matrix gene expression in cartilaginous neoplasms. Am J Pathol 1997, 150:2133-2141

7. Aigner T, Loos S, Müller S, Sandell LJ, Perris R, Unni KK, Kirchner T: Cell differentiation and matrix gene expression in mesenchymal chondrosarcomas. Am J Pathol 2000, 156:1327-1335

\section{The Function of COX-2 in Human Ovarian Carcinoma}

\section{To the Editor-in-Chief:}

Denkert et al ${ }^{1}$ conducted an elegant study demonstrating the pattern of COX-1 and COX-2 expression in ovarian cancer with important implications for ovarian cancer prevention and therapy. However, their contention that COX-2 is an independent prognostic factor is invalid, as several studies have shown that optimal surgical debulking and platinum-based chemotherapy are important independent prognostic variables in patients with ovarian cancer, ${ }^{2,3}$ and, unfortunately, data on patient treatment is lacking in Denkert et al's paper. ${ }^{1}$

Roshan Agarwal

The Institute of Cancer Research

London, United Kingdom

\section{References}

1. Denkert C, Köbel M, Pest S, Koch I, Berger S, Schwabe M, Siegert S Reles A, Klosterhalfen B, Hauptmann S: Expression of cyclooxygenase 2 is an independent prognostic factor in human ovarian carcinoma. Am J Pathol 2002, 160:893-903

2. Voest EE, van Houwelingen JC, Neijt JP: A meta-analysis of prognostic factors in advanced ovarian cancer with median survival and overall survival measured with the log (relative risk) as main objectives. Eur J Cancer Clin Oncol 1989, 28A:1328-1330

3. Hunter RW, Alexander NDE, Soutter WP: Meta-analysis of surgery in advanced ovarian carcinoma: is maximum cytoreductive surgery an independent determinant of prognosis? Am J Obstet Gynecol 1992, 166:504-511

\section{Author's Reply:}

The function of COX-2 in ovarian carcinoma is still the topic of an ongoing discussion in the scientific community. We thank Dr. Agarwal for his interest in our study ${ }^{1}$ and for his comments, which provide us with the opportunity to present some additional data that, we believe, will be helpful to answer the questions raised.

We completely agree that chemotherapy and the extent of surgical therapy are important prognostic factors in ovarian carcinoma that have been identified by several meta-analyses. $^{2-5}$ Based on these results, platinumbased chemotherapy as well as optimal surgical debulking are now established therapeutic strategies for treatment of ovarian carcinoma.

To investigate whether the expression of COX-2 is a prognostic parameter in the subgroup of patients receiving a platinum-based chemotherapy, we have updated our exploratory statistical analysis. Data on chemotherapy was available for $58(67 \%)$ of the 86 patients with invasive ovarian carcinoma. Of these patients, $81.4 \%$ received a platinum-based chemotherapy, $8.4 \%$ were treated with non-platinum chemotherapy and $10.2 \%$ did not receive any chemotherapy. In our study, the type of chemotherapy (platinum-based versus other versus none) was not a prognostic factor in univariate survival analysis. This is in line with the remark by Bristow ${ }^{3}$ that "survival comparisons of platinum- and non-platinum-treated patients are largely of historical interest," due to the fact that the majority of patients now receive platinum-based primary chemotherapy. Furthermore, the distribution of COX-2-positive and -negative cases was not significantly different in the different therapeutic groups. We performed an exploratory survival analysis for the subgroup 


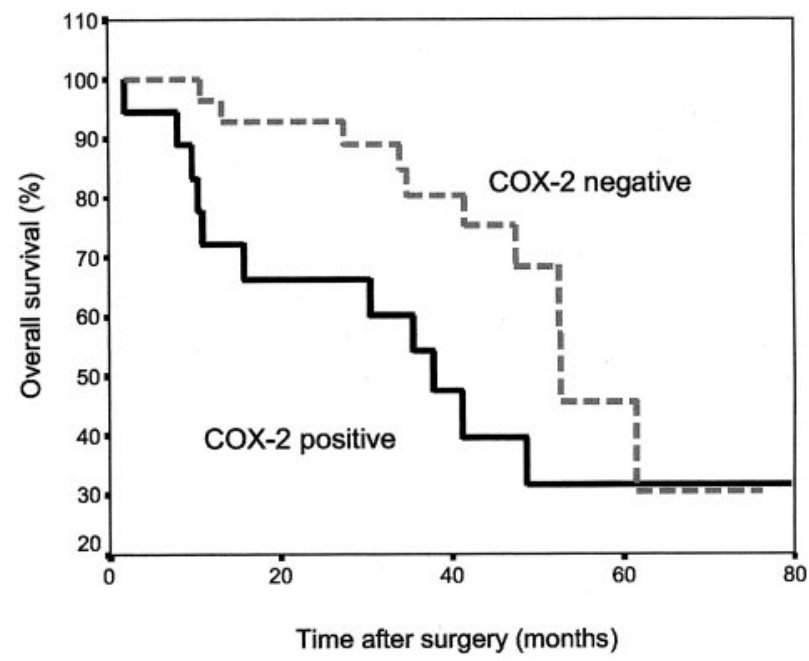

Figure 1. Univariate survival analysis (Kaplan-Meier) of 47 patients with ovarian carcinoma treated with a platinum-based chemotherapy: the median survival of patients with tumors positive for COX-2 was reduced compared to patients with tumors negative for COX-2 (log rank test, $P=0.03)$.

of patients that were treated with platinum-based chemotherapy. In this group of patients, the median survival of patients with tumors negative for COX-2 was 52.7 ( \pm 5.11) months, while it was reduced to 37.9 ( \pm 6.42$)$ months for patients with tumors positive for COX-2 (Figure $1, \log$ rank test, $P=0.03$ ). This suggests that COX-2 is a prognostic factor for patients with ovarian carcinomas receiving standard chemotherapy.

The majority of studies showing a prognostic effect of cytoreductive surgery restrict their investigation to FIGO stage III or IV cases. ${ }^{2-5}$ Thus, the therapeutic concept of extensive surgical de-bulking is relevant only for therapy of advanced ovarian carcinoma. Since we included 30\% of patients with stage I or II ovarian carcinomas in our study, cytoreductive therapy could not be separately assessed as an independent factor in multivariate analysis for the whole study sample. In the subgroup of patients treated with radical surgery, detailed data on the amount of residual tumor after surgery is currently available for 22 cases. Seventeen of these cases $(77.3 \%)$ had a residual tumor smaller than $2 \mathrm{~cm}$, while $5(22.7 \%)$ had more than $2-\mathrm{cm}$ residual tumor. In this small group of cases, there was no association between expression levels of COX-2 and the amount of residual tumor. We agree, however, that a more detailed and refined analysis studying the role of COX expression and different therapeutic regimens as well as their interaction should be performed in a larger set of patients with advanced ovarian carcinomas.

We appreciate Dr. Agarwal's comment that a multivariate analysis can only give information on the relationship between parameters that are actually included in this analysis. In our study, we have identified COX-2 as an independent prognostic parameter for overall survival with the parameters of FIGO stage, grade, histological type, as well as patient age included in the analysis. The number of possible variables in multivariate analysis is restricted by the number of cases investigated. Therefore, we cannot exclude that factors not analyzed might be associated with increased COX-2 expression as well as survival.
It has been pointed out in a recent commentary ${ }^{6}$ that our study as well as similar studies ${ }^{7}$ on the role of COX-2 in ovarian carcinoma should be seen as hypothesis-generating reports. Prospective and retrospective studies investigating a larger number of cases are needed to determine whether expression of COX-2 may be a clinically relevant prognostic and/or therapeutic marker.

\section{Carsten Denkert Steffen Hauptmann}

Charite Hospital

Humboldt University Berlin, Germany

\section{References}

1. Denkert C, Köbel M, Pest S, Koch I, Berger S, Schwabe M, Siegert S, Reles A, Klosterhalfen B, Hauptmann S: Expression of cyclooxygenase 2 is an independent prognostic factor in human ovarian carcinoma. Am J Pathol 2002, 160:893-903

2. Hoskins WJ, Bundy BN, Thigpen JT, Omura GA: The influence of cytoreductive surgery on recurrence-free interval and survival in small-volume stage III epithelial ovarian cancer: a gynecologic oncology group study. Gynecol Oncol 1992, 47:159-166

3. Bristow RE, Tomacruz RS, Armstrong DK, Trimble EL, Montz FJ: Survival effect of maximal cytoreductive surgery for advanced ovarian carcinoma during the platinum era: a meta-analysis. J Clin Oncol 2002, 20:1248-1259

4. Bertelsen K: Tumor reduction surgery and long-term survival in advanced ovarian cancer: a DACOVA study. Gynecol Oncol 1990 38:203-209

5. Piver MS, Lele SB, Marchetti DL, Baker TR, Tsukada Y, Emrich LJ: The impact of aggressive de-bulking surgery and cisplatin-based chemotherapy on progression-free survival in stage III and IV ovarian carcinoma. J Clin Oncol 1988, 6:983-989

6. Nasi ML, Castiglione M: Cyclooxygenase-2 (COX-2) a new prognostic and predictive factor for ovarian cancer? Are all the criteria fulfilled? Ann Oncol 2002, 13:1169-1171

7. Ferrandina G, Lauriola L, Zannoni GF, Fagotti A, Fanfani F, Legge F Maggiano N, Gessi M, Mancuso S, Ranelletti FO, Scambia G: Increased cyclooxygenase-2 (COX-2) expression is associated with chemotherapy resistance and outcome in ovarian cancer patients. Ann Oncol 2002, 13:1205-1211

\section{Rearranged Epstein-Barr Virus Genome in Hodgkin's Disease and Angioimmunoblastic Lymphadenopathy: Swiss Results}

\section{To the Editor-in-Chief:}

With special interest we studied the article titled, "A Defective, Rearranged Epstein-Barr Virus Genome in EBERNegative and EBER-Positive Hodgkin's Disease," by Gan and colleagues, ${ }^{1}$ published in the March 2002 issue of The American Journal of Pathology. The authors assessed the presence of the BamHI W/Z rearrangement, known to disrupt viral latency, ${ }^{2}$ in clinical samples of 56 American and Brazilian children and adolescents suffering from Hodgkin's disease (HD). Applying a sensitive PCR assay on DNA extracted from paraffin-embedded material, Gan et $\mathrm{al}^{1}$ detected the juxtaposition of the BamHI W and Z fragments in 10 of $32(31 \%)$ EBER-positive and in 8 of 24 EBER-negative HD tumors. 